\title{
$\mathbb{T} \mathbb{E} \mathbb{E} \mathbb{R A B A} \mathbb{R} \mathbb{H} O:$ \\ $D_{\mathbb{E} S \mathbb{A}} \mathbb{I O S} \quad \mathbb{E} \quad \mathbb{P}_{\mathbb{E}} \mathbb{R} \mathbb{P} \mathbb{E} \mathbb{C} \mathbb{T} \mathbb{V} \mathbb{S}^{\mathbb{1}}$ \\ Luiz Ojima Sakuda* \\ Flávio de Carvalho Vasconcelos**
}

RESUMO

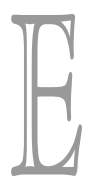

ste artigo discute o teletrabalho, definido amplamente como "o uso de computadores e telecomunicações para mudar a geografia do trabalho aceita", e que envolve diversos aspectos (econômico, social, cultural, organizacional, tecnológico, ambiental, legal e outros) e diversos atores (organizações, indivíduos, governos, fornecedores de tecnologias de informação, sindicatos e outros). Iniciamos com o contexto no qual o teletrabalho está inserido, o estado da pesquisa, e exploramos seus desafios nos níveis (i) individual, destacando a questão espaço-temporal, sua relação com os conflitos de valores e demandas profissionais e pessoais e o isolamento; (ii) organizacional, com as questões de comunicação, colaboração e unidade de propósito e os desafios da virtualidade e a inserção da gestão do conhecimento neste cenário; e (iii) global, discutimos a importância da localização, os limites e as potencialidades da tecnologia para a flexibilidade espacial. Finalmente, procuramos integrar estes desafios e apontar direções de pesquisa.

$\mathbb{A B S T R A C T}$

his article discusses telework, defined widely as "the use of computers and telecommunications to change the accepted geography of work", and involves several aspects (economic, social, cultural, organizational, ambient, legal, and others), social actors (organizations, individuals, governments, technology suppliers, unions, and others). Starting from the context of telework and status of research, the article explores the challenges on the several levels of study: (i) the individual, specially the space-time issue and its relationship with the conflicts of professional and personal values and demands, and isolation; (ii) organizational, regarding communication, collaboration and cohesion issues, as well as changes of the virtual and the insertion of knowledge management in this scenario; and (iii) global, when we discuss the importance of location, the limits and potential of technology for the space flexibility. Finally, the article tries to integrate these challenges and propose future research.

\footnotetext{
${ }^{1}$ Os autores agradecem as contribuições do revisor anônimo da O\&S. Uma versão preliminar deste artigo foi apresentada no CLADEA.

* Prof. FGV Management

** Prof. FGV-EAESP
} 
teletrabalho precisa ser compreendido no contexto de um amplo processo de transição, que tem um potencial diferente das outras revoluções: "uma mutação revolucionária para toda humanidade, mutação só comparável à invenção da ferramenta e da escrita, no labor das sociedades de classes, e que ultrapassa largamente a da revolução industrial do século XVIII" (Lojkine, 1995). Este autor mostra que a antinomia entre as teses 'pessimistas' sobre a 'desqualificação-superqualificação do trabalho' e as teses 'otimistas' sobre o progresso técnico e o 'fim da divisão do trabalho' pode realmente ser superada, a parir do momento em que as mutações sociotécnicas sejam tomadas com potencialidades contraditórias e não como conseqüências automáticas de uma evolução linear (p. 21).

Este artigo está dividido em 3 partes: (i) o contexto no qual o teletrabalho está inserido, o estado da pesquisa em teletrabalho e o teletrabalho no Brasil; (ii) os desafios do teletrabalho nos níveis individual, organizacional e global; e (iii) as considerações finais e sugestões para pesquisa futura.

\section{$\mathbb{T E} \mathbb{E} \mathbb{E} \mathbb{R A B A} \mathbb{R} \mathbb{H}$}

\section{Q QUE ER TELETRABALIHO}

A palavra telecommuting, que originou a palavra teletrabalho em português, foi cunhada pelo norte-americano Jack Nilles em The TelecommunicationsTransportation Trade Off, em 1976. Commuting é uma palavra que designa a viagem de ida e volta entre o domicílio e o local de trabalho. Esta abordagem para o teletrabalho é refletida na fase inicial da presença do tema em publicações acadêmicas de negócios, como em Lopez e Gray (1977). Com o desenvolvimento do campo, preocupações individuais e organizacionais passaram a ser hegemônicas. De um modo mais amplo, o teletrabalho é "o uso de computadores e telecomunicações para mudar a geografia do trabalho aceita" (European Commission, 2000), e envolve diversos aspectos (econômico, social, cultural, organizacional, tecnológico, ambiental, legal e outros) e diversos atores (organizações, indivíduos, governos, fornecedores de tecnologias de informação, sindicatos e outros).

Lamound, Daniels e Standen (1997) analisaram 900 artigos entre 1985 e 1996 contendo as palavras-chave teleworking, telecommuting ou homeworking, e observaram que a maioria da literatura em teletrabalho envolve prescrições baseadas principalmente na experiência de indivíduos e não utiliza teoria ou pesquisas recentes. O artigo destaca que os autores americanos costumam utilizar telecommuting e os europeus telework; o que reflete o foco de atenção das diversas definições desenvolvidas até hoje: localização (telecommuting, trabalho fora do escritório) e processo (teleworking, uso de tecnologias de informação e comunicação -TICs ou TIs). Os autores concluem que teletrabalho: (1) é um processo que envolve uma grande variedade de práticas; (2) que não existe uma forma de teletrabalho, e, como corolário, não existe a melhor forma de teletrabalho; e (3) é melhor entendido como um fenômeno multidimensional, com características que variam ao longo de cinco dimensões: (i) uso de TI, (ii) intensidade de conhecimento, (iii) contato intra-organizacional, (iv) contato extra-organizacional e (v) localização.

EITO (1988) consolida os aspectos abordados no campo do teletrabalho propondo classificações quanto ao contexto, parâmetros e modelos. EITO divide os modelos de teletrabalho em 7 categorias:

(1) Teletrabalho a domicílio

(2) Telecentro - subdividido em Centro administrado pelo empregador, Centro com várias empresas usuárias e Centro público ou semi-público local

(3) Escritório flexível/ hotelling/ hot desking 
(4) Móvel, independente do local

(5) Equipes multi-localizadas e/ou distribuídas

(6) Concentrativo

(7) Internacional/ off-shore.

EITO propõe também 8 categorias de parâmetros: (1) Arranjo do local de trabalho e "base" de trabalho percebida, (2) Relação de emprego, (3) Proporção de tempo no teletrabalho, (4) Extensão do uso da rede de comunicação eletrônica, (5) Grau de formalidade, (6) Grau de apoio, (7) Aceitação organizacional, ou grau de disseminação do teletrabalho na organização e (8) Grau de autonomia.

Combinando o trabalho de EITO com o de Lamound, Daniels e Standen, acreditamos ser possível abarcar todas as variáveis que podem ser estudadas, classificando o objeto de estudo conforme as dimensões, parâmetros e modelos.

O tema, além de ser recorrente na mídia, também despertou interesse de instituições internacionais como o International Labour Organization (Di Martino e Wirth, 1990) e foi tema direto ou indireto de eventos e documentos de discussão com a sociedade, como no Livro Verde da Sociedade de Informação brasileiro (SocInfo, 2000).

\section{$\mathbb{P}_{\text {esquisas }}$ e Publicaçöes sobre Teletraballio}

McCloskey e Igbaria (1998) fazem uma revisão da literatura empírica e argumentam que o pouco estudo empírico feito na área tem grandes limitações e contradições. Os autores distinguem cinco tipos:

- Estudos-piloto, úteis para uma análise exploratória, mas que não podem ser utilizados para o desenvolvimento de um modelo;

- Uso, que estabelece a extensão da participação do teletrabalho, focando em tipos de trabalho ou uma região geográfica, mas que sem o uso, definições conceituais comuns não possibilitam comparar os resultados dos diversos estudos;

- Valores e percepções, que apesar de prover importantes contribuições sobre os valores e atitudes dos teletrabalhadores, têm utilidade limitada, pois o fato dos empregados que nunca teletrabalharam acreditarem que o teletrabalho resulta em menor desenvolvimento da carreira, o que não quer dizer, necessariamente, que a carreira será retardada;

- Atitudes e resultados do trabalho, que tentam estabelecer vantagens e desvantagens do teletrabalho, mas ainda não foram conclusivas; e

- Questões sobre a família e o trabalho, que assim como o item anterior, é necessário um maior aprofundamento.

Estes autores consideram que o corpo da pesquisa existente é enfraquecido por três limitações básicas: a falta de uma definição formal de teletrabalho, fraqueza metodológica e a falta de controle em variáveis externas potencialmente significativas. Estas limitações geram resultados contraditórios e na impossibilidade de formar um corpo cumulativo de pesquisa em teletrabalho (Mccloskey e Igbaria:1988, p. 342).

O primeiro aspecto, a falta de conceitos comuns, é recorrente, agravado pelo fato de que o teletrabalho é explorado sob uma variedade de nomes, como flexplace, homework, flexwork, telework, telecommuting e outros. Os autores mostram que os estudos consideraram de modo diferenciado os aspectos de tecnologia, localização, relação empregatícia e estrutura, produzindo um conhecimento bastante fragmentado. O segundo aspecto, a fraqueza metodológica, se refere principalmente ao tamanho da amostra, muito pequeno, que pode ser útil para os estudos exploratórios, mas cujos resultados não podem ser generalizados. O terceiro aspecto, a falta de controle de fatores externos, é bastante relacionado com os aspectos anteriores. Muitas vezes, os teletrabalhadores são tratados como um grupo homogêneo, sem considerar as diferenças de relações empregatícias, tipo de trabalho e nível de participação do teletrabalho. 
Apesar da abrangência da pesquisa, estes autores não comentam o estudo ECATT - Electronic Commerce and Telework Trends in Europe (Empirica, 2000), que é a continuação de um esforço de pesquisa que começou em 1984 (Huws, Korte e Robinson, 1990) e continuou em 1994 com o TELDET - Telework Developments and Trends (Korte e Wynne, 1996). Este trabalho merece destaque pela visão histórica do desenvolvimento do teletrabalho, não apenas em termos quantitativos, mas também analisa aspectos qualitativos como percepções dos indivíduos e organizações sobre o teletrabalho. Outro trabalho que complementa esta visão histórica é EITO (1998), que aponta os fatores catalisadores e constrangedores do teletrabalho nos períodos que considera de início (1980-metade dos 1990), de desenvolvimento (atual) e de maturidade (depois de 2005).

O teletrabalho é o tema de eventos anuais, como a série do International Workshop on Telework, iniciada em 1996 pelo International Telework Foundation (atual International Telework Academy), realizada por um parceiro local em cada uma de suas edições, e deu origem a coletâneas sobre teletrabalho (Jackson e Van Der Wielen, 1997; Jackson, 1999 e Rapp e Jackson, 2003). A oitava edição foi realizada em São Paulo, com a co-realização do Conselho Regional de Administração de São Paulo. Além deste evento, vale destacar o European Assembly on Teleworking and New Ways of Working, organizado pela Comissão Européia, o Festival Européen du télétravail et des télé-activités, organizado pelo Systemia, e os encontros do ITAC International Telework Association \& Council. Para um aprofundamento do percurso acadêmico do teletrabalho, são representativos os artigos de Feldman e Gainey (1997), Shin, Sheng e Higa (2000), Baruch (2001) e Daniels, Lamound e Standen (2001), que contêm revisões bibliográficas e sugestões de agenda de pesquisa.

$\mathrm{Na}$ visão executiva, temos diversos artigos que identificam diversos paradoxos e que propõem formas de agir. Pearlson e Saunders (2001) destacam três: (1) aumento na flexibilidade e na estrutura, (2) maior individualidade, maior trabalho de grupo, e (3) maior responsabilidade, maior controle As autoras sugerem estratégias para os gestores, e enfatizam que aceitem o paradoxo, clareiem o ponto de vista, considerem o tempo e usem novas perspectivas, administrando o trabalho e não o trabalhador. Entre outros artigos com preocupação na implantação do teletrabalho, podemos destacar Apgar IV (1998), Watad e Disanzo (2000), Hartman, Stoner e Arora (1992) e Maruca (1998).

Além de artigos em publicações executivas, o teletrabalho tem sido popularizado por livros e websites voltados a gerentes e teletrabalhadores. No Brasil, foram traduzidos Kugelmass (1996), Nilles (1997) e Bawa e Dubash (1998), nos quais os autores destacam procedimentos para a implantação do teletrabalho, exemplos e tabelas de funções mais adequadas ao teletrabalho, conforme uma série de características das tarefas de cada um, e modelos de contrato e de avaliação de programas de teletrabalho. Na Internet, o destaque é o website de Gil Gordon, consultor que participou, em 2002, do evento anual da Associação Brasileira de Recursos Humanos (ABRH).

No Brasil, o trabalho acadêmico pioneiro foi o de Soares (1995) que segue uma tradição crítica de estudo do trabalho a domicílio (como Abreu e Sorj, 1993) e do trabalho computacional de baixa especialização (como Pearson e Mitter, 1993). Outros trabalhos sobre o tema incluem: Oliveira (1996), Andreassi (1997), Mello (1997, 1999), Troppe (1998), Sakuda (1999, 2001), Mello e Sakuda (2000), Ferreira Jr (2000), Steil e Barcia (2001), Silva (2001) Macedar e Reinhard (2002), Hanashiro e Dias (2002) e Costa (2004).

\section{Os Desafios Do TELETRABALIIO}

\section{O Desafio Individidul}

Antes da revolução industrial, o trabalho a domicílio era feito por trabalhadores que detinham os meios de produção e trabalhavam com seus próprios mé- 
todos, incluindo o uso do tempo. O teletrabalho pode levar o trabalhador e o trabalho para muitos lugares, inclusive de volta ao domicílio. Os valores sociais predominantes, no entanto, continuam a ser os da sociedade industrial. Neste sentido, trabalhos como o de Greenhaus e Beutell (1985), que examinam o conflito entre os papéis sociais exigidos pelo trabalho e pela família; e de Lambert (1990), que estuda o processo que relaciona estas duas dimensões da vida, podem contribuir bastante para o debate. Na sociedade industrial, boa parte do trabalho não era passível de ser feita fora do escritório, que tem horários rígidos. Com a flexibilização temporal e espacial, a atividade produtiva poderá regular toda a vida do indivíduo, não importando o local e o horário.

Jean-François Chanlat coordenou uma série marcante sobre as dimensões esquecidas nas organizações. A questão espaço-temporal foi abordada em cinco artigos: Hassard (1996), Fischer (1996), Chanlat (1996), Gasparini (1996) e Kamdem (1996). Destes, destacaremos o trabalho de Hassard.

Para Hassard, a sociologia industrial tende a ignorar que muitos sistemas não funcionam no ritmo das máquinas, no paradigma linear-quantitativo; e cita como exemplos a venda, o marketing, o desenvolvimento de produtos, as profissões liberais e os serviços de emergência. O paradigma linear-quantitativo tem como valores o realismo, o determinismo, a linearidade, a homogeneidade, o nomológico e a quantidade. Em contraste, o paradigma cíclico-qualitativo valoriza o nominalismo, o voluntarismo, a circularidade, a heterogeneidade, o ideográfico e a qualidade.

O desafio é tentar integrar estes dois paradigmas no estudo do impacto desta mudança na relação do indivíduo com o seu trabalho, em um ambiente em que não será possível separar as dimensões do trabalho e da vida privada como feito durante a era industrial. Brocklehurst (1999) discute poder e identidade no novo trabalho tecnológico a domicílio e destaca que a reestruturação de tempo e espaço do trabalho não libertou os indivíduos das regras e recursos do local de trabalho, mas causou ansiedade àqueles que trabalharam para recriar as mesmas regras. O autor descreve a dificuldade de auto-identificação dos teletrabalhadores, lembra que as formas tradicionais de identidade de trabalho estão ligadas à distinção entre masculino-provedor-público e feminino-dependente-privado, e sugere que é prematuro olhar as novas formas de organização do trabalho como benéficas ou calamitosas.

Vega e Brennan (1996) discutem o isolamento a partir de diversas perspectivas - comunicação, economia, tecnologia, psicologia, psicologia social e sociologia - e relacionam 13 fatores organizacionais (controle de limites externo, integração com a comunidade, cultura organizacional, significado do papel do trabalho, grau de poder, compartilhamento de referências, normas e valores do grupo, autoridade, interação colegiada, feedback, artefatos de status e interação face-a-face) com situações objetivas e subjetivas de isolamento social; e sugerem que o teletrabalho pode aumentar a propensão a sentimentos subjetivos de isolamento social.

Em uma abordagem mercadológica, podemos também estudar como o teletrabalho pode mudar a estrutura da rotina de trabalho e lazer, seus hábitos de consumo e outros aspectos da vida pessoal, procurando novas oportunidades de negócio (Chevron e Primeau, 1996).

O DEsAfiO ORGANiZACIONAL

Em 1938, Barnard (1971, p.101) já destacava a importância da comunicação nas organizações: uma organização "passa a existir quando (1) há pessoas aptas a comunicarem entre si (2) que estão desejando contribuir com sua ação (3) para servir um propósito comum". Baseado neste conceito, o autor traça três funções executivas essenciais: prover o sistema de comunicação, promover a garantia de esforços essenciais e formular e definir propósito. As três funções são claramente interdependentes, e as novas formas de organização e trabalho exigem mudanças significativas. O sistema de comunicação formal e informal é claramente dife- 
rente em uma organização que utiliza o teletrabalho intensamente; a formação de uma comunidade de propósito em um cenário com organizações em rede, onde nem todas as informações e objetivos são compartilhados permanentemente. Um aspecto importante destes desafios é a gestão do conhecimento.

Nonaka e Takeuchi (1995) consideram o conhecimento como a unidade básica de análise para explicar o comportamento das organizações. Raghruam (1996) aborda este tema, destacando o caráter linear das comunicações feitas por e-mails e voice-mails em contraste com a complexidade das comunicações diretas e coletivas do local de trabalho tradicional, especialmente da comunicação informal; e levanta outros aspectos do relacionamento entre os pares. Por exemplo, a convivência próxima e constante propicia condições mais favoráveis à criação, fortalecimento e manutenção de relações de confiança do que as relações virtuais; e a confiança é um dos fatores fundamentais para a disseminação do conhecimento tácito. O artigo conclui com o alerta de que, sem os cuidados necessários, o teletrabalho pode acarretar perda de conhecimento tácito e explícito da organização.

O teletrabalho pode ser estudado também no contexto da organização virtual. É dentro deste contexto que temos edições ou sessões especiais no Organizational Science (Desancrits e Monge, 1999) e em outros periódicos (Igbaria, 1998; Narasimhan e Higa, 1996).

Handy (1995) prevê que, se existir um escritório do futuro, será mais como um clube: um lugar para se reunir, comer, comemorar, com lugares para atividades e não para pessoas. Este autor destaca que, paradoxalmente, quanto mais virtual a organização for, maior a necessidade dos indivíduos de se encontrarem pessoalmente. Destaca a importância da confiança na organização virtual, e sugere que a afiliação deverá mudar de natureza: ao invés de pertencer a um lugar, os membros irão pertencer a uma comunidade. Neste sentido, a discussão sobre a criação e a dinâmica de comunidades virtuais (Rheingold, 1993 e Wellman et alli,1996) normalmente focada em nível social, poderá ter uma contribuição para o nível organizacional importante, assim como estudos como o de Kurland e Egan (1999), que discutem a relação da percepção de justiça dos teletrabalhadores.

O Desafio Giobal

Em seu modelo de vantagem competitiva das nações, Porter (1993) traz o modelo do "diamante" para a análise da competitividade de indústrias nacionais, cujos atributos são: (i) condições de fatores, (ii) condições de demanda, (iii) indústrias correlatas e de apoio, e (iv) estratégia, estrutura e rivalidade das empresas. Além destes determinantes fortemente inter-relacionados, o autor destaca a importância do acaso e o papel do governo. Da análise da dinâmica do modelo, vale ressaltar dois aspectos: o agrupamento de indústrias competitivas e o papel da concentração geográfica. O autor lista alguns exemplos de mecanismos que facilitam o intercâmbio dentro dos grupos, como o fluxo de informações e a coordenação, que criam confiança e diminuem as diferenças entre as empresas.

Entre os facilitadores do fluxo de informação, Porter (1993) cita as relações pessoais resultantes de freqüência à mesma escola, serviço militar, as ligações através da comunidade científica ou associações profissionais, os laços comunitários devidos à proximidade geográfica, as associações de comércio abrangendo grupos; e as normas de comportamento, como a crença na continuidade e nas relações de longo prazo. Como exemplos de fontes de congruência ou compatibilidade dentro dos grupos, o autor coloca os laços de família ou semelhantes entre empresas, a propriedade comum dentro de um grupo industrial, a propriedade de interesses acionários parciais, as diretorias interligadas e o patriotismo nacional.

A concentração geográfica de uma indústria, além de estimular a competitividade, poderá atrair talentos especializados, empresas derivadas e o interesse da universidade. Entre os exemplos que ilustram o modelo, o caso da indústria de azulejos italianos é particularmente interessante para nossa discus- 
são, pois mostra como redes de pequenas unidades de negócios, muitas das quais familiares e baseadas em trabalho a domicílio, podem ser competitivas. Goddard e Richardson (1996) defendem que as condições geográficas continuarão sendo muito importantes para o emprego de longo prazo, mesmo com a viabilidade de comunicação instantânea das TIs.

Esta discussão é particularmente interessante para o teletrabalho, pois mostra as limitações do jargão "trabalhar em qualquer lugar, a qualquer hora". A produção sobre empresas multinacionais poderá contribuir bastante nesta discussão. Por exemplo, Boudreau et alli (1998) destacam o uso da TI para aumentar a competitividade da empresa, de modo a superar barreiras temporais e espaciais de empregados e parceiros de negócios. Já Ghemawatt (2001) mostra que existem diversas dimensões de distância (cultural, administrativa, geográfica e econômica) que afetam de forma diferenciada as indústrias. Assim, podemos esperar diversas formas e velocidades diferentes de expansão do teletrabalho nas empresas, conforme suas próprias características: as de sua indústria e as de seus colaboradores, fornecedores e clientes.

\section{CONSIDERAÇÕES FINAIS}

Procuramos expor o contexto do teletrabalho, seus diversos aspectos e atores envolvidos, explorando os seus desafios nos níveis individual, organizacional e global. Acreditamos que o teletrabalho é um componente importante das mudanças na sociedade cujo avanço das TIs pode proporcionar. Estas mudanças têm potencialidades contraditórias, que podem ter conseqüências benéficas ou prejudiciais para os diversos atores envolvidos. As mudanças para os trabalhadores de baixa qualificação, especialmente aqueles localizados em regiões e países de baixo grau de desenvolvimento e que não têm possibilidades de mudança física (por meios legais) de migrar para outros países, têm uma forma de teletrabalho diferente dos trabalhadores de alta qualificação, com possibilidade de migrar internacionalmente e entre empresas e setores econômicos. Assim, a TI pode proporcionar flexibilidade real para um grupo, enquanto que para outro grupo poderá haver apenas um aumento de controle por conta da automatização. O teletrabalho em telecentros tem características diferentes do teletrabalho a domicílio, um programa corporativo com alto apoio organizacional, treinamento e suporte para os teletrabalhadores e seus gerentes terá uma dinâmica diferente de um programa conduzido informalmente, com a tolerância (e sem o apoio) da empresa. As vantagens percebidas pelos indivíduos dependem de seu momento de vida, tempo e distância de commuting e outros fatores, assim como para as organizações.

O teletrabalho tem áreas de sobreposição com dois campos de estudo e prática mais desenvolvidos do que o teletrabalho: a educação à distância e o comércio eletrônico. No Brasil, pesquisadores destas duas áreas poderiam aproveitar o acesso às empresas e às pessoas, além de certa familiaridade com o tema, para expandir o foco e auxiliar a expansão de suas fronteiras. Além de estudos quantitativos, são também necessários estudos jurídicos e parcerias com entidades acadêmicas e não-acadêmicas, nacionais e internacionais; e utilizar o teletrabalho o máximo possível para a própria pesquisa.

$\mathbb{R} \mathbb{E} \mathbb{E} \mathbb{R} \hat{E} \mathbb{N} C \mathbb{A} S$

ABREU, Alice Rangel de Paiva, SORJ, Bila (orgs). Trabalho Invisível. Estudos sobre trabalhadores a domicílio no Brasil. Rio de Janeiro: Rio Fundo, 1993.

ANDREASSI, Tales. Virtualização das organizações: o caso do teletrabalho em uma consultoria. Revista de Administração da USP, v.4, n.4. p.77-83, 1997. 
APGAR IV, Mahlon. The Alternative Workplace: Changing Where and How People Work. Harvard Business Review, v.76, i.3, p.121-136, May-June, 1998.

BARNARD, Chester. As funções do executivo. São Paulo: Atlas, 1971.

BARUCH, Yehuda. The status of research on teleworking and an agenda for future research. International Journal of Management Reviews, v.3, i.2, p.113129, Jun 2001

BAWA, Joanna e DUBASH, Manek. O escritório em casa. São Paulo: Summus, 1998.

BOUDREAU, Marie-Claude; LOCH, Karen D.; ROBEY, Daniel; STRAUD, Detmar. Going Global: Using information technology to advance the compertitiveness of the virtual transnational organization. Academy of Management Executive, v.12, i.4, p.120-128, Nov 1998.

BROCKLEHERST, Michael. Power, Identity and New Technology Homework: Implications for 'New Forms' of Organizing". Organization Studies, v.22, i.3, p.445-466, 2001.

CHANLAT, Jean-François. "O Ser humano, um ser espacial e temporal" IN CHANLAT, Jean-François (Coord) O Indivíduo na Organização: dimensões esquecidas. São Paulo: Atlas, 1996.

CHEVRON, Jacques; PRIMEAI, Margaret. The telecommuting innovation opportunity. Journal of Consumer Marketing, v.13, n.4, p.40-48.

COSTA, Isabel de Sá Affonso da. Teletrabalho: Subjugação e Construção de Subjetividades IN Anais do XXVIII Encontro da ANPAD. Curitiba: ANPAD, 2004

DANIELS, Kevin; LAMOND, David; STANDEN, Peter. Teleworking: Frameworks for Organizational Research. Journal of Management Studies, v.38, i.8, p.11511185.

DESANTICS, Gerardine; MONGE, Peter. Introduction to Special Issue:

Communication Process for Virtual Organizations. Organization Science, v.10, i.6, Special Issue: Communication Process for the Virtual Organizations, p.693703, 1999.

DIMARTINO, Vittorio; WIRTH, Linda. Telework: A new way of working and living. International Labour Review, v.129, i.5, p.529-554, 1990.

EITO Telework: Status, Development and Issues IN EITO (Org) European Information Technology Observatory 98. Frankfurk: EITO - European Information Technology Observatory, 1998.

EMPIRICA. Electronic Commerce and Telework Trends (ECATT) - Final Report. Bonn: Empirica, 2000.

EUROPEAN COMMISSION e-Work: Status Report on New Methods to Work in the Information Society. Bruxelas: Office for Official Publications of the European Communities, 2000.

FELDMAN, Daniel C.; GAINEY, Thomas W. Patterns of Telecommuting and their consequences: framing the research agenda. Human Resource Management Review, v.7, i.4, p.369-388, 1997.

FERREIRA JR, José C. Telecommuting: O paradigma de um novo estilo de trabaIho. RAE Light, v.7, n.3, p.8-17, 2000.

FISHER, Gustave Nicholas. Espaço Identidade e Organização IN CHANLAT, JeanFrançois (Coord) O Indivíduo na Organização: dimensões esquecidas. São Paulo: Atlas, 1996. 
GASPARINI, Giovanni. Tempo e Trabalho no Ocidente IN CHANLAT, Jean-François (Coord) O Indivíduo na Organização: dimensões esquecidas. São Paulo: Atlas, 1996.

GHEMAWAT, Pankaj. Distance Still Matters: the Hard Reality of Global Expansion. Harvard Business Review, v.79, i.8, p.137-146, September, 2001.

GODARD, Donald e RICHARDSON, Ranard. Why Geography Still Matter: What Jobs Go Where? IN DUTTON, William (Org). Information and Communication Technologies: Visions and Realities. Nova York: Oxford University Press, 1996

GREENHAUS, Jeffrey H.; BEUTELL, Nicholas J. Sources of Conflict Between Work and Family Roles. Academy of Management Review, v.10, n.1, p.76-88, 1995

HANASHIRO, Darcy Mitiko Mori; DIAS, Wellington Fonseca Dias. O Sistema de Teletrabalho: Algumas Implicações de um Ambiente Virtual IN Anais do XXVI Encontro da ANPAD, Salvador: ANPAD, 2002.

HANDY, Charles. Trust and the Virtual Organization. Harvard Business Review, v.73, i.3, p.40-50, May-June, 1995

HARTMAN, Richard; STONER, Charles R.; ARORA, Raj. Developing Successful Organizational Telecommuting Arrangements: Worker Perceptions and Managerial Prescriptions. S.A.M. Advanced Management Journal, v.57, i.3, 1992

HASSARD, John. Tempo de Trabalho: outra dimensão esquecida nas organizações. IN CHANLAT, Jean-François (Coord) O Indivíduo na Organização: dimensões esquecidas. São Paulo: Atlas, 1996.

HUWS, Ursula; KORTE, Werner B.; ROBINSON, Simon. Telework: Towards the Elusive Office. Wiley and Sons, Chichester et al, 1990.

IGBARIA, Magid. Special Section: Managing Virtual Workplaces and Teleworking with Information Technology. Journal of Management Information Systems, v.14, i.4, p.5-6, 1998.

JACKSON, Paul James; VAN DER WIELEN, Jos (Eds.). Teleworking: New International Perspectives from Telecommuting to the Virtual Organisation. Londres: Routledge, 1997.

JACKSON, Paul James (Ed). Virtual Working: Social and Organizational Dynamics. Londres: Routledge, 1999.

KAMDEM, Emmanuel. "Tempo e Trabalho na África" IN Chanlat, Jean-François (Coord) O Indivíduo na Organização: dimensões esquecidas. São Paulo: Atlas, 1996.

KORTE, Werner B.; WYNNE, Richard. Telework: penetration, potential and practice in Europe. Amsterdam: IOS Press, 1996.

KUGELMASS, Joel. Teletrabalho: Novas Oportunidades para o Trabalho Flexível: seleção de funcionários, benefícios e desafios, novas tecnologias de comunicação. São Paulo: Atlas, 1996.

KURLAND, Nancy B; EGAN, Terri D. Telecommuting: Justice and Control in the Virtual Organization. Organization Science, v.10, i.4, p.500-513, 1999.

LAMBERT, Susan J. Process Linking Work and Family: A Critical Review and Research Agenda. Human Relations, v.43, i.3, p.239-257, 1990.

LAMOND, David; DANIELS, Kevin; STANDEN, Peter. Defining Telework: What Is It Exactly? IN JACKSON, P.; der WIELEN, J. (orgs) Second International Workshop on Telework "Building Actions on Ideas". Tilburg: Work and Organizational Research Centre/ Brunel University, 1997. 
LOJKINE, Jean. A Revolução Informacional. São Paulo: Cortez, 1995.

LOPEZ, David; GRAY, Paul. The Substitution of Communication for Transportation - a Case Study. Management Science, v.23, i.11, p.1149-1169, 1977.

MARUCA, Regina Fazio. How do you manage an off-site team? Harvard Business Review, v.76, i.4, p.22-35, July-August, 1998.

MACADAR, Marie Anne; Reinhard, Nicolau. Telecentros Comunitários Possibilitando a Inclusão Digital: um Estudo de Caso Comparativo de Iniciativas Brasileiras. IN Anais do XXVI Encontro da ANPAD, Salvador: ANPAD, 2002.

MCCLOSKEY, Donna Weaver; IGBARIA, Magid. A Review of the Empirical Research on Telecommuting and Directions for Future Research. IN IGBARIA, Magid.; TAN, Margaret The Virtual Workplace. Londres: Idea Group, 1998.

MELLO, Álvaro. Avolta para casa: desmistificando o telecommuting! Alvaro Mello, São Paulo, 1997.

MELLO, Álvaro. Teletrabalho (telework): o trabalho em qualquer lugar e a qualquer hora. Rio de Janeiro : Qualitymark, ABRH Nacional, 1999.

MELLO, Álvaro e SAKUDA, Luiz Ojima. Telework Trends in Brazil: preliminary research results. IN RAPP, Birger, JACKSON, Paul James (Eds). 2000 and beyond: Teleworking and the Future of Work? Proceedings from the $5^{\text {th }}$ International Workshop on Telework. Estocolmo: International Telework Foundation, 2000.

NARASIMHAN, Sridhar; HIGA, Kunihiko. Introduction to the Special Issue on Telework. Journal of Organizational Computing and Electronic Commerce, v.6, i.4, p.325-326, 1996.

NILLES, Jack M. Fazendo do Teletrabalho uma realidade: um guia para telegerentes e teletrabalhadores. São Paulo: Futura, 1997.

NONAKA, Ikujiro, TACHEUCHI, Nobuko. The knowledge-creating company: how Japanese companies create the dynamics of innovation. Nova York: Oxford University Press, 1995.

OLIVEIRA, Martha Maria Veras. Ergonomia e o teletrabalho a domicílio. Dissertação de Mestrado. Florianópolis: Universidade Federal de Santa Catarina, 1997.

PEARLSON, Keri E.; SAUNDERS, Carol S. There's no place like home: Managing telecommuting paradoxes. Academy of Management Executive, v.15, i.2, p.117129, 2001.

PEARSON, Ruth; MITTER, Swasti. Employment and working conditions of lowskilled information-processing workers in less developed countries.

International Labour Review, v.132, i.1, p. 49-65, 1993.

PORTER, Michael. A Vantagem Competitiva das Nações. São Paulo: Campus, 1993.

RAGHURAM, Sumita. Knowledge creation in the telework context. International Journal of Technology Management, Special Issue on Unlearning and Learning for Technological Innovation, v.11, i.7/8, p.859-870, 1996.

RAPP, Birger.; JACKSON, Paul James. (Eds) (2003). Organisation and Work Beyond 2000. Heidelberg: Physica-Verlag, 2003.

RHEINGOLD, Howard. The Virtual Community: Homesteading on the Electronic Frontier. HarperPerennial, 1993. 
SAKUDA, Luiz Ojima. Business Centres and Virtual Offices to Telework in Brazil IN VASCONCELOS, Marcos Augusto; CORRÊA, Henrique; CSILLAG, João Mário; DELÁZARO Filho, José; SALINAS, Adriana Vieira Anais do II SIMPOI - II Simpósio de Administração da Produção, Logística e Operações Industriais. São Paulo: FGV-EAESP, 1999.

SAKUDA, Luiz Ojima. Teletrabalho: Desafios e Perspectivas. Dissertação de Mestrado. São Paulo: FGV-EAESP, 2001.

SHIN, Bongsik; LIU SHENG, Olivia R.; HIGA, Kunihiko. Telework: Existing Research and Future Directions. Journal of Organizational Computing \& Electronic Commerce, v.10, i.2, p. 85-101, 2000.

SILVA, Ronaldo André Rodrigues da. Telecomutters: Desconstrução e Reconstrução dos Conceitos de Subjetividade no Mundo do Trabalho. Anais do XXV Encontro da ANPAD. Campinas: ANPAD, 2001.

SOARES, Angelo. Teletrabalho e Comunicação em Grandes CPDs. Revista de Administração de Empresas, v.35, n.2, p.64-77, 1995.

SOCINFO Livro Verde. Brasília: Programa da Sociedade da Informação do Ministério da Ciência e Tecnologia - Sociedade da Informação no Brasil:SOCINFO, 2000.

STEIL, Andrea V.; BARCIA, Ricardo M. Um Modelo para análise da prontidão organizacional para implantar o teletrabalho. Revista de Administração da USP, v. 36, n.1, p.74-84, 2001.

TROPPE, Alberto. Organizações Virtuais e Teletrabalho. Qualitymark: Rio de Janeiro, 1998.

VEGA, Gina; BRENNAN, Louis Isolation and technology: the human disconnect. Journal of Organizational Change Management, v.13, i.5, p.468-481, 2000.

WATAD, Maohmoed M; DISANZO, Frank J. Case Study: The Synergism of Telecommuting and Office Automation. Sloan Management Review, Winter 2000, p.85-96, 2000.

WELLMAN, Barry; SALAFF, Janet; DIMITROVA, Dimitriva; GARTON, Laura; GULIA, Milena. Computer Networks as Social Networks: Collaborative Work, Telework, and Virtual Community. Annual Review of Sociology, v.2, p.213-238, 1996. 